\title{
The Ensemble Interpretation of Quantum Mechanics and Scientific Realism
}

\author{
Alexander Pechenkin \\ Faculty of Philosophy \\ Lomonosov Moscow State University \\ Moscow GSP-1, Russia \\ S. Vavilov Institute for the History of Science and Technology of RAS \\ Baltiiskaia ul., 14 \\ Moscow, Russia \\ Email:a_pechenk@yahoo.com
}

Abstract: The article takes under consideration three versions of the ensemble (statistical) interpretation of quantum mechanics and discusses the interconnection of these interpretations with the philosophy of science. To emphasize the specifics of the problem of interpretation of quantum mechanics in the USSR, the Marxist ideology is taken into account.

The present paper continues the author's previous analysis of ensemble interpretations which emerged in the USA and USSR in the first half of the 2oth century. The author emphasizes that the ensemble approach turned out to be a dead end for the development of the interpretation of quantum mechanics in Russia. The article also argues that in Soviet Russia, the classical Copenhagen (standard) approach to quantum mechanics was used. The Copenhagen approach was developed by Lev Landau in 1919-1931 and became the basis of the Landau-Lifshitz famous course on quantum mechanics, one of the classics of twentieth-century physics literature (the first edition was published in 1947).

Although Vladimir A. Fock's approach to the interpretation of quantum mechanics differs from the standard presentation by Lev Landau and Evgeny Lifshitz, Fock put forward a very important principle that complementarity is a "firmly established law of nature".

The fundamental writings of Lev Landau, Vladimir Fock and Igor Tamm, the authors of the mid-twentieth century, did a lot to defend the standard point of view such as the popular interpretations by Landau and Lifshitz. This approach can be traced back to Landau's early writings and to Fock's criticism of the ensemble approach. 
Keywords: Copenhagen interpretation, hidden variables, Marxism, measurement, positivism, uncertainty relations

The present author's 2012 paper and book published in 2019 (Pechenkin, 2012; 2019) took under consideration the interpretations of quantum mechanics which were put forward in the 1930s and a while later. The author came to the conclusion that, in contrast with Central Europe, the majority of US physicists and a considerable fraction of physicists in the Soviet Union proclaimed the ensemble (statistical) version of the interpretation of quantum mechanics. Physicists and philosophers in Central Europe focused on the Copenhagen interpretation of quantum theory and on the problems which this interpretation presented (mainly on the problems of reduction of the wave packet and on the issues of hidden variables).

This does not mean that there was a kind of Chinese wall between the philosophy of quantum mechanics in Central Europe and the rest of the world. As is well known, the ensemble interpretation proceeded from Albert Einstein's speech at the Fifth Solvay Congress and the subsequent philosophical meditations of Karl Popper (1934-1935). In the Soviet Union, the authoritative figures spoke in favor of the ensemble interpretation (L. I. Mandelstam, K. V. Nikolskii, B. M. Hessen). However, Fock, Landau, and Tamm spoke (with some reservations) in favor of the Copenhagen interpretation.

The present paper is historical in nature. It shall not discuss the controversial philosophical issue: the interconnection between the philosophical beliefs and speculations concerning quantum mechanics. The first section is dedicated to Landau's early contribution to the Copenhagen interpretation of quantum mechanics, the second section is concerned with Karl Popper's contribution to the philosophy of quantum mechanics, the third section is dedicated to the contribution of the Soviet physicist Dmitrii Blokhintsev, and the fourth section is dedicated to the Canadian Leslie Ballentine. Blokhintsev and Ballentine spoke in favor of the ensemble approach. 


\section{Landau and Lifshitz as proponents of the Copenhagen interpretation}

Lev Landau spent the years 1929-1931 abroad. He worked in Germany, Switzerland, England, and especially in Copenhagen, where Niels Bohr became his supervisor. In 1929, Landau began cooperating with Rudolf Peierls, who started as an assistant of Wolfgang Pauli.

Landau and Peierls contributed to the quantum theory of measurements by considering predictable measurements.

Having demonstrated that the existence of predictable measurements, that is, measurements which assure that for every possible measurement result there exists a state of the system in which this measurement yields with certainty the result obtained, does not imply the existence of reproducible measurements, that is, measurements which assure that a repeated performance yields the same result, Landau and Peierls showed that the state of the system after the measurement is not necessarily identical with the state associated with the obtained measurement result. "Cognizant of this fact, they pointed out that the time-energy relation affirms that this difference of states leads to an energy indeterminacy of the order of magnitude $h \boldsymbol{\nabla} t$, so that within a time interval $\boldsymbol{\nabla} t$ no measurement can be performed for which the energy indeterminacy is less than $h \boldsymbol{\nabla} t$." (Jammer, 1974, p. 143).

To some extent, the discussion between Mandelstam and Tamm, on the one side, and V. A. Fock and his graduate student N. S. Krylov, on the other, can be treated as a continuation of the Landau-Peierls' above analysis. In his 1942-1943 note on energy in quantum mechanics, Mandelstam objected to Landau-Peierls' interpretation of the energy-time uncertainty relation. In turn, this article was taken under criticism by Fock in his paper written in collaboration with his former student N. S. Krylov (for details see Pechenkin, 2019). 


\section{Karl Popper's contribution to the foundations of quantum mechanics}

In his book on the history of the philosophy of quantum mechanics, Max Jammer called Popper "one of the great human thinkers of our time" (Jammer, 1974, p. 174). In this book, Jammer attentively followed how Popper argued with the great authorities in physics by pushing forward his version of the ensemble interpretation of quantum mechanics. He criticized the "subjective interpretation which reads the more precisely the position of a particle is measured then less is known about its momentum and vice versa" (Jammer, 1974, p. 448). Jammer writes,

Popper advocated what he called the statistical objective interpretation. [...] Popper always held that the problems to which quantum mechanics applicable are essentially statistical problems and as such require statistical answers. According to Popper the vectors of Hilbert space provide statistical assertion from which no predicative inference can be drawn for individual particles. (Jammer, 1974, p. 448)

Jammer emphasized that Popper was influenced by Einstein's speech at the Fifth Solvay Congress (1927). “According to viewpoint I," Einstein declared,

the de Broglie-Schrödinger waves do not represent one individual particle but rather an ensemble of particles distributed in space. [...] According to viewpoint II, quantum mechanics is considered as a complete theory of individual processes; each particle moving toward the screen is described as a wave packet which after diffraction, arrives at a certain point of the screen and $|\psi(r)|^{2}$ expresses the probability that at a given moment one and the same particle shows its presence at $r$. (Jammer, 1974)

Popper, however, published his meditations on quantum mechanics seven years later, in his paper in Naturwissenschaften (1934) and in his book Logik der Forschung (1935, according to the translator's note it was published in autumn 1934).

The main target of Popper's criticism was Bohr's complementarity principle and Heisenberg's interpretation of the uncertainty principle, the interpretation presented in Heisenberg's Chicago Lectures. Popper emphasized that Heisenberg's uncertainty relations can be considered as a theorem of quantum theory. However, Heisenberg's interpretation of the uncertainty relations does not belong to the 
unavoidable conclusions of quantum theory. Popper advocated what he called the statistical objective interpretation of the uncertainty relations.

Given an ensemble of particles (aggregate of particles or sequence of experiments provided with one particle which after each experiment is reprepared in its original state) from which at a certain moment and with given precision $\Delta \mathrm{x}$ those having a certain position are selected; the moments $p$ of the latter will then show a random scattering with the range of scatter $\Delta p$ where $\Delta x \Delta p \geq h$ and vice versa (Jammer, 1974, p. 176).

In his 1934-1935 papers and book, Popper did not use the terms 'realism' and 'scientific realism'. This terminology was not applicable in his The Logic of Scientific Discovery (1959), published in English, either. Popper's problem was to formulate the objective interpretation which allowed us to treat quantum mechanics as a scientific theory that did not depend on metaphysics.

Popper turned to the concept of realism in his Postscript to The Logic of Scientific Discovery (first volume, 1975). His reference to realism had not been essential for his treatment of the structure of uncertainty relations. However, by treating quantum mechanics from the realist position, Popper outlined the implicit context of his criticism of indeterminist metaphysics. "The task of science," Popper writes in his Postscript,

which I have suggested is to find a satisfactory explanation can hardly be understood if we are not realists. For a satisfactory explanation is one which is not ad hoc, and this idea — the idea of independent evidence — can hardly be understood without the idea of discovery of progressing to deeper levels of explanation, without the idea that there is something for us to discover, and something for us to discuss critically. (Jammer, 1974, p. 145)

Popper's following comment is already concerned with his 1934 attack on the Bohr-Heisenberg approach:

It may perhaps $[\ldots]$ be mentioned that my interpretation of Heisenberg's indeterminacy formulae as scatter relations was both an attempt to criticize Heisenberg's Machian positivism ("observables") and to eliminate what I regarded as his metaphysical dogmatism: his theory that indeterminacy formulae indicated the limit of scientific knowledge (Jammer, 1974, p. 181).

In the third volume of his Postscript, Quantum Theory and the Schism in Physics, Popper developed the propensity interpretation of probability, the 
interpretation which helps him to criticize again the Bohr-Heisenberg approach to quantum mechanics. The propensity interpretation, however, requests a special consideration.

\section{Blokhnintsev's ensemble interpretation}

Dmitrii Ivanovich Blokhintsev (1908-1975) is one the great figures of Soviet science. Blokhintsev presented his $\mathrm{PhD}$ thesis in 1935; his scientific supervisor was Igor E. Tamm, one of the great theoreticians in the Soviet Union, who received a Nobel Prize for the discovery and explanation of the Cherenkov effect (together with Ilya Frank and Pavel Cherenkov).

Blokhintsev became a professor at Lomonosov Moscow State University in 1936. In the 1950s, he was appointed as Chair of Nuclear Physics at the Department of Physics. He received the title of the corresponding member of the Soviet Academy of Sciences.

He was an organizer and first director of the Physics and Energy Laboratory in Obninsk (1947-1956), one of the secret establishments of Soviet nuclear endeavors. This laboratory was later transformed into a research institute. Blokhintsev was the organizer and director of the Joint Institute for Nuclear Research in Dubna (1956-1965). The idea of this institute was to gather outstanding physicists and young talented specialists from 11 socialist countries (in contemporary terminology they could be named the "satellites" of the Soviet Union) to work together.

In 1976, Blokhintsev received the medal of the Hero of Socialist Labor, the highest state award in the Soviet Union.

There is, however, a blank spot in Blokhintsev's scientific biography. In spite of his achievements and positions, he was not elected Academician. This fact is evidence of that there was something specific in his relations with the higher establishment of the Soviet Academy of Sciences.

In 1944, Blokhintsev's textbook Introduction to Quantum Mechanics was published. Jammer calls it "the first comprehensive anniversary textbook on quantum mechanics in the Russian language" (Jammer, 1974, p. 445). This book was written "in the spirit of Heisenberg's interpretation according to which the wave function represents the man's knowledge of the state rather than the state of 
the system itself" (Jammer, 1974, p. 445). Five years later, Blokhintsev published a revised edition, titled Foundations of Quantum Mechanics, "which by virtue of its excellent didactic approach became one of the most popular textbooks ever on quantum mechanics in the Russian language" (Jammer, 1974, p. 445). This book went to five editions and was reprinted many times (the fifth edition was issued in 1975). Besides Foundations of Quantum Mechanics, Blokhintsev published a philosophical book Principal Issues of Quantum Mechanics (1966).

In contrast to Introduction to Quantum Mechanics, Blokhintsev's second book, Foundations of Quantum Mechanics had an articulated anti-Copenhagen context. "The chapter which concerns the concept of state in quantum mechanics has been drastically changed and the idealistic conceptions of quantum mechanics which are now widespread abroad are subjected to criticism". (Blokhintsev, 1975)

In his 1949 book, Blokhintsev emphasized the concept of quantum ensembles. According to him, by knowing the wave function we can provide statistical predictions only.

As a result, a single measurement is not informative: it only shows whether an unlikely or highly likely event has occurred. Only the distribution of a considerable amount of measurements has an objective character. Generally speaking, in quantum mechanics, we cannot reproduce the experiment with a particle. Measurement can change the state of a particle. This means that an experiment in quantum mechanics implies a considerable amount of particles which are placed in the same circumstance independently of each other (the experiment implies a quantum ensemble). (Blokhintsev, 1949, p. 59)

It should be noted that Blokhintsev published not only scientific papers and books. In 1951, his paper which had an articulated political tenor was published in the Soviet leading journal on physics. Probably this paper was Blokhintsev's reaction to the new ideological company, established by the 1946 Communist Party's decision on journals Zaria and Leningrad - the decision which attacked the great Russian poet Anna Akhmatova. Communist ideologists of various scale and activists immediately joined this company, speaking out with exposures of the writers and scientists who could be treated as carriers of bourgeois views and showing political vigilance (for details see Loren Graham, Alexander Vucinich and other historians of science).

Some fragments of Blokhintsev's 1951 paper look like an ordinary review of the writings on the interpretation of quantum mechanics. Blokhintsev proclaimed his 
ensemble approach and attacked the Copenhagen interpretation. However, this paper contains references to the main ideological documents of the Communist Party: to Lenin's Materialism and Empirio-criticism and to the fragment of the The History of $\operatorname{VKP}(b)$, written by Stalin. The main point was to announce that the Copenhagen interpretation of quantum mechanics (the Bohr-Heisenberg interpretation) is inconsistent with materialism as a philosophical position.

In 1959, Blokhintsev published the article 'Lenin's book Materialism and Empirio-criticism and the modern conceptions of the structure of the elementary particles', where he continued, in more soft expressions, the ideological line announced in his 1951 paper.

Blokhintsev, like other Soviet scientists and philosophers, never called himself "a realist" or "a scientific realist". He called himself "a materialist" or "dialectical materialist". However by criticizing the Copenhagen interpretation, Blokhintsev was close to the position which was announced as "realism" in the literature on the philosophy of science in the last decades of the 20th century (this was "realism" as an antipode of both "instrumentalism" and "empirical constructivism"). As a realist, he insisted that a theory in physics should be treated as representative of reality which is something external with respect of the theory. However, like a dialectical materialist, he treated the scientific concepts as reflections on reality, he emphasized that quantity tends to be transformed into quality, and insisted that the concepts need to be treated as "in development".

As a matter of fact, Blokhintsev reduced dialectical materialism to a number of trivialities. However, his references to Marx, Engels, Lenin, and Stalin supported the division of the university teachers to proclaim scholasticism as it is represented by the "laws" and "categories" of dialectics.

In the 1975 version of his Foundations of Quantum Mechanics, Blokhintsev kept a more quiet tone. He wrote in its 'Preface' that the hard ideological struggle about the conceptual problems of quantum mechanics belonged to the past (Blohkintsev, 1975, p. 11).

As was noted above, Blokhintsev proceeded from the ensemble interpretation of quantum mechanics. "In this book, quantum mechanics was presented from the point of view of quantum ensembles. In contrast to the Copenhagen interpretation this position emphasizes the objective character of quantum ensembles and the corresponding regulations and gives a more modest role to the observer." (Blokhintsev, 1975, p. 616) 
"The conception of quantum ensembles," Blokhintsev writes,

is very close to Gibbs' classical conception. Within the Gibbs' framework a microsystem is treated in the interaction with the macroscopic thermostat $M$ with the temperature $\theta$. The probability $W(P, Q)$ of a result of the measurement of dynamic variables of the microsystem $(P, Q)$ is related to the ensemble which was formed by the unrestricted reproduction of the situations consisting of the microsysyem $\mu$ and the thermostat $M$; in other words - by the unrestricted reproduction of the system $\mu$ in the same macroscopic situation determined by the thermostat having the temperature $\Theta$. As a result the probability $\mathrm{W}(P, Q)$ characterizes both the micro system $(\mathrm{P}, \mathrm{Q})$ and its macroscopic situation-the temperature of thermostat $\theta$. In accordance with the Gibbs' classical ensemble, the quantum ensemble is constituted by an unrestricted reproduction of the situations given by the micro system $\mu$ (but not its single copy!) included into identical macroscopic situation. (Blokhintsev, 1975, p. 617)

By comparing the quantum ensembles with Gibbs' classical ensembles, Blokhintsev implicitly followed the US physicists John Slater, Edwin C. Kemble and some other classics of the ensemble interpretation of quantum mechanics. In some of his papers, Soviet physicist L. I. Mandelstam also referred to Gibbs' ensembles. The quantum ensemble of Gibbs' conception of an ensemble represents the collection of many repetitions of the same experiment, agreeing as concerns the large scale macroscopic properties which we can control but taking different values of microscopic properties.

In his book on the philosophy of quantum mechanics (1965, English translation in 1968), Blokhintsev formulated the following argument in favor of the ensemble interpretation:

If the wave function is a characteristic of a single particle it would be of interest to perform such a measurement on a single particle (say, an electron) which would allow us to determine its own individual wave function. No such measurement is possible. (Blokhintsev, 1968, p. 50)

This argument was reproduced by one of the active proponents of ensemble approach, Leslie Ballentine (see the following section).

M. A. Markov, who has critically examined Blokhintsev's interpretation of quantum mechanics, pointed to several wage formulations which Blokhintsev admitted in his books (Markov, 2010). One of the wage formulations was cited above. What does it mean- "a more modest role of the observer"? 
Blokhintsev's attitude to hidden variables is unclear, too. As he wrote, the question of hidden variables is a matter of faith. It is possible to find arguments for the hidden variables (this was the attitude of Einstein (1949)) but there are many arguments to reject this conception.

\section{Leslie E. Ballentine: The ensemble interpretation and empiricism}

Let us cite Leslie Ballentine, who became the main ideologist of the ensemble approach to quantum mechanics in the post-Second World War years. Ballentine is a professor (now emeritus) at Simon Fraser University in Canada. Like Blokhintsev, Ballentine published a textbook on quantum mechanics, the textbook which went to several editions $(1990,2015)$, and a number of papers on the philosophy of physics.

Ballentine clearly distinguishes between two main interpretations of quantum mechanics.

The statistical interpretation according to which a pure state (and hence a general state) provides a description of certain statistical properties of an ensemble of similarly prepared systems, but need not to provide a complete description of an individual system. This interpretation is upheld by Einstein, Popper, and Blokhintsev. (Ballentine, 1970, p. 360)

Ballentine also writes about the "interpretations which assert that a pure state provides a complete description" of an individual system (e.g., an electron). This class contains a great verity of members, from Schrödinger's original attempt to identify the electron with the wave packet solution of his equation to the several versions of the Copenhagen interpretation" (Ballentine, 1970, p. 360).

In contrast to Blokhintsev, Ballentine was definite with respect to hidden variables.

The statistical interpretation which regards quantum states as being descriptive of ensembles of similarly prepared systems, is completely open with respect of hidden variables. It does not demand them, but it makes the search for them entirely reasonable (this was the attitude of Einstein). (Ballentine, 1970, p. 374) 
Ballentine attentively describes the measurement process. As is well known, this is one the difficult points of the interpretation of quantum mechanics.

The discussion of the analysis of measurement according to the statistical interpretation was so simple and natural that further comment almost seems redundant. But if instead of the basic assumption of the statistical interpretation that a state vector characterizes an ensemble of similarly prepared systems, that a state provides a complete description of an individual system, then the situation is quite different. (Ballentine, 1970, p. 368)

The problems which arose in the standard theory of measurement are well known. This led to the controversial concept of the reduction of the wave packet and to the frankly subjective interpretations according to which the quantum state description is not supposed to express the properties of a physical system or an ensemble of systems but our knowledge of these properties and changes of state are identified with the changes of knowledge.

Ballentine does not treat his interpretation of quantum mechanics as an argument for scientific realism. This is a "sound interpretation using minimal assumptions" (Ballentine, 1970, p. 358).

Specialists in the philosophy of science treat his position as an argument in favor of empiricist philosophy. "This is my view," Hooker writes, "that this doctrine pretty clearly represents a strategy of emasculating by fiat the semantic content of quantum mechanics until it says only what is compatible with empiricism" (Hooker, 1987, p. 390). Bas C. van Fraassen (1995, pp. 298-299), who put forward the philosophy of constructive empiricism, also sympathizes with Ballentine's ensemble interpretation.

However, Ballentine's analysis of the problem of measurement can be placed in the controversy "realism-subjectivism" and gives arguments in favor of realism.

\section{Conclusion}

As was mentioned at the beginning, this article is historical in nature. It demonstrates the philosophical contexts which historically justified the ensemble interpretation of quantum mechanics. Conventionally, if one is not inclined to provide the sophisticated differences, this context can be characterized as scientific realism. 
It should be noted that the ensemble interpretation is currently not popular. The Stanford Encyclopedia of Philosophy does not contain an article 'Ensemble (statistical) interpretation' although it contains articles 'Copenhagen interpretation of quantum mechanics', 'Many-worlds interpretation of quantum mechanics', and 'Modal interpretations on quantum mechanics'.

In 2001, the Russian journal Physics Uspekhi, or Uspekhi Fizicheskikh Nauk, provided the discussion surrounding M. B. Menskii's paper 'Quantum mechanics: new experiments, new applications, new problems'. Seven papers (presumably philosophical) were published to express reaction to Menskii's paper, however no references were made to the ensemble interpretation which was popular in Russia in the 20th century (Mandelstam, Nikolskii, Hessen, Blokhintsev, and others) in these papers.

The present article is also evidence of the crisis of the ensemble approach in quantum mechanics in the 21 st century.

\section{References}

Ballentine, L. E. (1970), 'The statistical interpretation of quantum mechanics,' Reviews of Modern Physics, vol. 42, pp. 358-380. https://doi.org/10.1103/RevModPhys.42.358

Ballentine, L. E. (1990), Quantum Mechanics, Englewood Cliffs, NJ: Prentice Hall International Inc.

Ballentine, L. E. (2015), Quantum Mechanics. A Modern Development, New Jersey: World Scientific. https://doi.org/10.1142/9038

Blokhintsev, D. I. (1944), Vvedenie v kvantovuiu mekhaniku [Introduction to quantum mechanics], Moscow \& Leningrad: GITTL.

Blokhintsev, D. I. (1949), Osnovy kvantovoi mekhaniki [Foundations of quantum mechanics], Moscow \& Leningrad: GITTL.

Blokhintsev, D. I. (1951), 'Kritika idealisticheskogo ponimania kvantovoi mekhaniki' [The criticism of the idealistic understanding of quantum theory], Usp. Fiz. Nauk, vol. XLV, no. 2, pp. 195-228.

Blokhintsev, D. I. (1966), Printsipialnye voprosy kvantovoi mekhaniki [The principal problems of quantum mechanics], Moscow: Nauka.

Blokhintsev, D. I. (1975), Osnovy kvantovoi mekhaniki [Foundations of quantum mechanics], 5th ed., Moscow: Nauka.

Hooker, C. A. (1987), A Realistic Theory of Science, New York: State Univ. of New York Press. 
Jammer, M. (1974), The Philosophy of Quantum Mechanics, New York: Wiley.

Mandelstam, L. \& Tamm, I. (1945), 'The uncertainty relation between energy and time in non-relativistic quantum mechanics,' The USSR Journal of Physics, vol. 9, pp. 249-254.

Markov, M. A. (2010), Tri interpretatsii kvantovoi mekhaniki [Three interpretations of quantum mechanics], 2nd ed., Moscow: URSS.

Pechenkin, A. (2012), 'The early ensemble interpretations of quantum mechanics in the USSR and USA,' in Studies in the History and Philosophy of Modern Physics, vol. 1, pp. 2-11. https://doi.org/10.1016/j.shpsb.2011.11.006

Pechenkin, A. (2019), L. I. Mandelstam and His School in Physics, 2nd ed., Cham: Springer. https://doi.org/10.1007/978-3-030-17685-3

van Fraassen, B. C. (1995), Quantum Mechanics. An Empiricist View, Oxford: Oxford University Press.

Alexander Pechenkin graduated from the Mendeleev Institute of Chemistry and Technology (Faculty of Organic Substances) and Lomonosov Moscow State University (Faculty of Mechanics and Mathematics). He received his PhD from the Institute of Philosophy of the Academy of Sciences in 1968. He is a professor of Moscow State University and lectures courses in the history and philosophy of science. He also works for the S. Vavilov Institute for the History of Science and Technology of the Russian Academy of Sciences. 